\title{
Convergence Analysis for Wave Equation by Explicit Finite Difference Equation with Drichlet and Neumann Boundary Condition
}

\author{
Kedir Nebi Habib \\ Department of Mathematics, College of Natural Science, Arba Minch University, Arba Minch, Ethiopia
}

Email address:

Kedirnebi@gmail.com

\section{To cite this article:}

Kedir Nebi Habib. Convergence Analysis for Wave Equation by Explicit Finite Difference Equation with Drichlet and Neumann Boundary Condition. Mathematics Letters. Vol. 7, No. 2, 2021, pp. 19-24. doi: 10.11648/j.ml.20210702.11

Received: October 24, 2020; Accepted: May 3, 2021; Published: May 26, 2021

\begin{abstract}
There are many problems in the field of science, engineering and technology which can be solved by differential equations formulation. The wave equation is a second order linear hyperbolic partial differential equation that describes the propagation of variety of waves, such as sound or water waves. In this paper we consider the convergence analysis of the explicit schemes for solving one dimensional, time-dependent wave equation with Drichlet and Neumann boundary condition. Taylor's series expansion is used to expand the finite difference approximations in the explicit scheme. We present the derivation of the schemes and develop a computer program to implement it We use spectral radius of Matrix obtained from discretization and Von Neumann stability condition to determine stability, and consistence of the method from truncated error from discretized method. Using Lax Equivalence Theorem, convergence of the methods was described by testing consistency and stability of the methods. And it is found out that the scheme is stable with the Drichlet boundary and conditionally stable with Derivative boundary condition.
\end{abstract}

Keywords: Wave Equation, Explicit Method, Convergence, Stability

\section{Introduction}

The wave equation is a second order linear hyperbolic partial differential equation that describes the propagation of variety of waves, such as sound or water waves. It arises in different fields such as an acoustics, electromagnetic or fluid dynamics $[6,7]$. In many situations finding analytic solutions to partial differential equation is unrealistic or even impossible. Numerical methods that utilize computer algorithms are then used to find approximate solution $[2,3$, 10]. The focus of this paper is to determine the stability and convergence of finite difference schemes that approximates a solution of wave equation. Suppose that an elastic string of length $\mathrm{L}$ is tightly stretched between two supports at the same horizontal level. So that the $\mathrm{x}$-axis lies along the string. The elastic string may be thought of as violin sting, guy wire or possibly an electric power line. Suppose that the string is set in motion so it vibrates in vertical plane and let $u(x, t)$ denote the vertical displacement experienced by the string at the point $x$ at time $t$ if damping effects, such as air resistance are neglected. If the amplitude of the motion is not too large, then $u(x, t)$ satisfies the equation. $u_{t t}=c^{2} u_{x x}$ On domain $0<x<L, t>0$ the equation is one dimensional wave equation. [11]

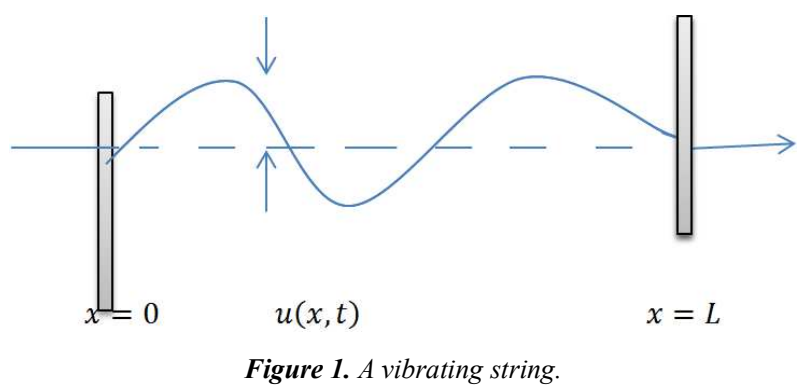

The coefficient $c^{2}$ in the equation is $c^{2}=\frac{T}{m}$ where $\mathrm{T}$ is the tension (force) in the string and $m$ is the mass per unit length of the string material. To describes the motion of the string completely it is necessary also to specify suitable initial and boundary conditions for displacement $u(x, t)$. The ends are assumed to remain fixed and therefore the boundary condition are $u(0, t)=0 u(L, t)=0 t \geq 0$ since the wave 
equation is of second order with respect to $t$ is plausible to prescribe two initial conditions those are the initial position of the string $u(x, 0)=f(x)$.

$0 \leq x \leq L$ And its initial velocity $u_{t}(x, 0)=g(x) 0 \leq$ $x \leq L$ where $f$ and $g$ are given functions. The finite difference methods are the techniques for numerical solution to the wave equation by the discretization of space and time

Let the string in the deformed state coincide with the interval $[0, L]$ on the $\mathrm{x}$ axis, and let $u(x ; t)$ be the displacement at time $t$ in the y direction of a point initially at $x$. the displacement function $u$ is governed by the mathematical model

$$
\frac{\partial^{2} u}{\partial t^{2}}=c^{2} \frac{\partial^{2} u}{\partial x^{2}}+f(x, t) x \in(0, L), t \in(0, T)
$$

(Governing equation)

$$
\begin{aligned}
& u(x, 0)=f(x), \frac{\partial u(x, 0)}{\partial t}=g(x) x \in(0, L) \text { (Initial condition) } \\
& u(0, t)=u(L, t)=0 \text { (Boundary conditions) } t \in(0, T)
\end{aligned}
$$

Since this PDE Contains a second-order derivative in time, we need two initial conditions, here $u(x, 0)=$ $f(x)$ specifying the initial shape of the string, $f(x)$, and $\frac{\partial u(x, 0)}{\partial t}=g(x)$ In addition, PDEs need boundary conditions, here $u(0, t)=u(L, t)=0$, specifying that the string is fixed at the ends, that ist the Displacement $u$ is zero. The solution $u(x, t)$ varies in space and time and describes waves that are moving with velocity $c$ to the left and right.

\section{Finite Difference Methods}

The finite difference techniques are based up on the approximations that permit replacing differential equation by finite difference equation. There finite difference approximations are algebraic in form, and the solutions are related to grid points. Thus, a finite difference solution basically involves three steps:

Dividing the solution into grids of notes.

Approximating the given differential equation by finite difference equivalence that relates the solutions to grid points.

Solving the difference equations subject to the prescribed boundary conditions and or Initial conditions. [2, 5, 9]

\subsection{Explicit Finite Difference Method}

The numerical solution of one dimensional wave equation using explicit scheme is obtained and the error calculated. To determine the stability and convergence, we will consider the simplified form of the wave equation (1) Common form.

$c^{2} \frac{\partial^{2} u}{\partial x^{2}}=\frac{\partial^{2} u}{\partial t^{2}}+f(x, t)$ With the boundary condition $u(0, t)=u(L, t)=0$ And the initial condition $u(x, 0)$ $=f(x)$, and $\boldsymbol{u}_{\boldsymbol{t}}(x, 0)=g(x)$. Using finite difference we have

$$
\frac{\partial^{2} u}{\partial x^{2}}=\frac{u_{j+1, n}-2 u_{j, n}+u_{j-1, n}}{h^{2}}
$$

$$
\frac{\partial^{2} u}{\partial t^{2}}=\frac{u_{j, n+1}-2 u_{j, n}+u_{j, n-1}}{k^{2}}
$$

Substituting equations (4) and (5) into the equation (1) it is approximated by

$$
\begin{gathered}
c^{2} \frac{u_{j+1, n}-2 u_{j, n}+u_{j-1, n}}{h^{2}}=\frac{u_{j, n+1}-2 u_{j, n}+u_{j, n-1}}{k^{2}} \\
\frac{u_{j, n+1}-2 u_{j, n}+u_{j, n-1}}{k^{2}}=c^{2} \frac{u_{j+1, n}-2 u_{j, n}+u_{j-1, n}}{h^{2}} \\
u_{j, n+1}=2 u_{j, n}-u_{j, n-1}+\frac{c^{2} k^{2}}{h^{2}}\left(u_{j+1, n}-2 u_{j, n}+u_{j-1, n}\right)
\end{gathered}
$$

Making $u_{j, n+1}$ the subject and substituting $r^{2}=\frac{c^{2} k^{2}}{h^{2}}$ we obtain

$$
u_{j, n+1}=\left(2-2 r^{2}\right) u_{j, n}+r^{2} u_{j+1, n}+r^{2} u_{j-1, n}-u_{j, n-1}
$$

\subsection{Matrix Form of Explicit Scheme}

Referring to equation (1), we discretize in space, using $n$ nodes the temperature at time $\mathrm{j} \Delta t i$ given by $[\bar{u} \mathrm{j}=$ $u 2, \mathrm{n}, u 3, \mathrm{n}, u 4, \mathrm{n}, \ldots u \mathrm{n}-1, \mathrm{n}]^{T}$ since $u 1, j=0$ for all $\mathrm{j}$ When grouped in values in rows and using $\mathrm{r}=\frac{a k}{h}$ Equation (9) can be rearranged to obtain

$$
\bar{u}_{n+1}=r^{2} u_{j-1, n}+\left(2-2 r^{2}\right) u_{j, n}+r^{2} u_{j+1, n}-u_{j, n-1}
$$

The parameter $r=\frac{a k}{h}>0$ depends up on wave speed and the ratio of space and time step size the boundary condition (1.) Require that (2) this allow us to rewrite the system in matrix form

$$
\begin{aligned}
& \bar{u}_{n+1}=B \bar{u}_{n}-\bar{u}_{n-1}+b_{i}
\end{aligned}
$$

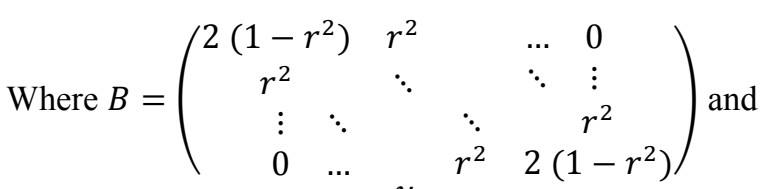

$$
\begin{aligned}
& \bar{u}_{n}=\left(\begin{array}{c}
u_{1, n} \\
\vdots \\
u_{j-1, n}
\end{array}\right)
\end{aligned}
$$

\subsection{One Dimensional Wave Equation with Derivative Boundary Condition}

The boundary condition $u(x, t)=f(x)$ makes $u$ change sign at the boundary, while the condition $u_{x}(x, t)=$ 0 perfectly reflects the wave. Our next task is to determine the stability with boundary condition $u_{x}(x, t)=0$, which is more complicated to express numerically. We shall present two methods for implementing $u_{x}(x, t)=0$ in a finite difference scheme, one based on deriving a modified stencil at the boundary, and another one bead on extending the mesh with ghost cells and ghost points.

Neumann boundary condition:

$$
\frac{\partial u}{\partial n}=n \cdot \nabla u=0
$$

The derivative $\frac{\partial u}{\partial n}$ is in the outward normal direction from a general boundary. 
For one dimension domain $[0 ; L]$, we have that:

$$
\left.\frac{\partial u}{\partial n}\right|_{x=L}=\frac{\partial}{\partial x},\left.\frac{\partial u}{\partial n}\right|_{x=0}=-\frac{\partial}{\partial x}
$$

Boundary conditions that specify the value of $u_{n}$ are known as Neumann conditions, while refer to specifications of $u$. When $\left(\frac{\partial u}{\partial n}=0\right.$ or $\left.u=0\right)$ it is Dirichler boundary condition

The Discretization of derivatives at the boundary in the finite difference scheme is used central differences in all the other approximations to derivatives in the scheme, it is tempting to implement (12) at $x=0$ and $t=t_{n}$ by the difference

$$
\frac{u_{-1, n}-u_{1, n}}{2 \Delta x}=0
$$

The problem is that $u_{-1, n}$ is not a $u$ value that is being computed since the point is outside the mesh. However, if we combine (13) with the scheme for $\mathrm{j}=0$,

\subsection{Fourier Method (Von Neumann Stability)}

Fourier stability analysis allows determining appropriate step sizes for an accurate solution when the wavelength or decay constant (which is given in terms of parameters such as a diffusion constant or wave velocity in the (PDE) has a certain value. Fourier stability analysis does not take boundary conditions for a specific problem into account. And it performed by substituting the analytic solution to a partial differential equation into the numerical finite difference equation. [8, 12-14]

Assume $u(x, t)=\sum_{k} \hat{u}(\mathrm{t}) \exp (\mathrm{ikx})$

The sum is over $k$, the Fourier frequencies. Now take for $u$ just one Fourier

Term $u(x, t)=\hat{u}(\mathrm{t}) \exp (\mathrm{ikx})$ And evaluate it at $\left(x_{j}, t_{n}\right)$ to get

$u_{j, n}=\hat{u}_{n} \exp (\mathrm{iki} \Delta \mathrm{x}), u_{j-1, n}=\hat{u}_{n} \exp (\mathrm{ik}(\mathrm{j}-1) \Delta \mathrm{x})$, $u_{j+1, n+1}=\hat{u}_{n+1} \exp (\mathrm{ik}(\mathrm{j}+1) \Delta \mathrm{x}) \quad$ and $\quad u_{j, n-1}=$ $\hat{u}_{n-1} \exp (\mathrm{ik}(\mathrm{j}+1) \Delta \mathrm{x})$ These expressions can be plugged directly into any finite difference scheme to check for stability. The growth rate $G$ is defined as $G=\left|\frac{\widehat{u}_{n+1}}{\widehat{u}_{n}}\right|$ for stability we need $\mathrm{G}<1$ for all frequencies $\mathrm{k}$. Conditional stability means we only have stability on a certain condition. Usually the condition limits $\Delta \mathrm{t}$ in function of $\Delta \mathrm{x}$

\subsection{Matrix Method to Determine Stability}

The condition for stability of methods is determined by finding the spectral radiu which is $p(A)=\max \left(\lambda_{i}\right)$ where $\lambda_{i}$ is an eigenvalue of matrix $A$, as illustrated below.

(i) If $p(A)<1$ then the system is stable.

(ii) If $p(A)<1$ then the system is stable.

(iii) If $p(A)>1$ then the system is unstable.

Tridiagonal matrices are often found in connection with finite differences. Tridiagonal matrices are easy to deal with since there exists ancient numerical methods both for solving their linear systems of equations and eigenvalue problem. Here we consider the eigenvalue problem for a general tridiagonal matrix of the form $[1,4,13,15]$

Lax Equivalence theorem: The Lax-Richtmyer Equivalence Theorem is often called the Fundamental Theorem of Numerical Analysis, even though it is only applicable to the small subset of linear numerical methods for well-posed, linear partial di erential equations. Along with Dahl Quist's equivalence theorem for ordinary di erential equations, the notion that the relationship consistency + stability $\Rightarrow$ convergence always holds has caused a great deal of confusion in the numerical analysis of di erential equations. In the case of PDEs, mathematicians are most often interested in nonlinear phenomena, for which LaxRichtmyer does not apply. More damningly, the forward implication that

$$
\text { Consistent }+ \text { stability } \Rightarrow \text { convergence }
$$

Theorem: Gerschgorin's theorem: Consider a square matrix $\mathrm{A}=\left(a_{i j}\right)$, for row $\mathrm{i}$ the disk $D_{i}$ have centre $a_{i i}$ and $\operatorname{radius} \sum_{\substack{j=1 \\ j \neq i}}^{N}\left|a_{i j}\right|$. Then the theorem states that;

Every eigenvalue of A lies in some $D_{i}$.

If $\mathrm{S}$ is the union of $\mathrm{s}$ disks $D_{i}$ such that $\mathrm{S}$ is disjoint from all other disks of this type, then $\mathrm{S}$ contains precisely $\mathrm{m}$ eigenvalues of $\mathrm{A}$.

\section{Analysis of Convergence for Explicit Scheme}

\subsection{Consistence of the Explicit Method}

The truncation error of the approximation of the time derivative is $T_{1}$ given by

$$
\frac{\partial^{2} u}{\partial t^{2}}=\frac{u_{j, n+1}-2 u_{j, n}+u_{j, n-1}}{k^{2}}+T_{1} \text { Where } T_{1}=-\frac{k^{2}}{12} \frac{\partial^{4} u}{\partial t^{4}}(x, \eta)
$$

Where $\mathrm{t}-k<\eta<t+k$ from the Taylor series expansion Similarly, for the space derivative, $\frac{\partial^{2} u}{\partial x^{2}}=\frac{u_{j+1, n}-2 u_{j, n}+u_{j-1, j}}{h^{2}}$ $+T_{2}$

Where $T_{2}=-\frac{h^{2}}{12} u_{x x x x}(\xi, t)$ is the truncation error $x-h \leq$ $\xi \leq x+h$ Combining the two terms above $\frac{\partial^{2} u}{\partial t^{2}}-a^{2} \frac{\partial^{2} u}{\partial x^{2}}=0$

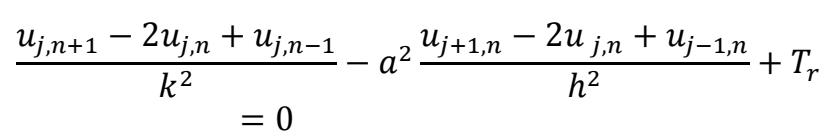

Where $T_{r}$ the truncation error $T_{r}=T_{1}-T_{2}=-\frac{k^{2}}{12} u_{t t t t}(x, \eta)$ $+\frac{h^{2}}{12} u_{x x x x}(\xi, t)$

Where $x-h \leq \xi \leq x+\mathrm{h}$ and $\mathrm{t}-k<\eta<t+k$ It then follows that

$$
\left|T_{r}\right| \leq \frac{k^{2}}{12} M_{t t t t}+\frac{h^{2}}{12} M_{x x x x}=\frac{1}{12}\left(k^{2} M_{t t t t}+h^{2} M_{x x x x}\right)
$$

Where $M_{t t t t}$ is a bound for $\left|u_{t t t t}(x, \eta)\right|$ and $M_{x x x x}$ is a bound for $\left|u_{x x x x}(\xi, t)\right|$ 
Hence $T_{r} \rightarrow 0$ as $\mathrm{k}, \mathrm{h} \rightarrow 0$ that is the difference approximation is consistent

\subsection{Stability Analysis of Explicit Method}

\subsubsection{Stability For Explicit with Drichlet Boundary \\ Condition}

The stability of this numerical scheme proceeds as follows

$u_{j, n+1}=\left(2-2 r^{2}\right) u_{j, n}+r^{2} u_{j+1, n}+r^{2} u_{j-1, n}-u_{j, n-1}$

We first need to recast the second order iteration system (14) in to a fist order system

$$
\begin{gathered}
\bar{u}_{n+1}=B \bar{u}_{n}-\bar{u}_{n-1}+b_{i} \\
\left(\begin{array}{c}
\bar{u}_{n+1} \\
\bar{u}_{n}
\end{array}\right)=\left(\begin{array}{cc}
B & -I \\
I & 0
\end{array}\right)\left(\begin{array}{c}
\bar{u}_{n} \\
\bar{u}_{n-1}
\end{array}\right)+b_{i}
\end{gathered}
$$

Let $\quad \bar{Z}_{n+1}=\left(\begin{array}{c}\bar{u}_{n+1} \\ \bar{u}_{n}\end{array}\right), \bar{Z}_{n}=\left(\begin{array}{c}\bar{u}_{n} \\ \bar{u}_{n-1}\end{array}\right)$ and $C=\left(\begin{array}{cc}B & -I \\ I & 0\end{array}\right)$ Implies that

$$
\bar{Z}_{n+1}=C \bar{Z}_{n}+b_{i}
$$

Therefore the stability of the method will be determined by the Eigen value of the coefficient matrix $\mathrm{C}$ the Eigen vector equation $C z=\lambda z$ where $z=\left(\begin{array}{l}u \\ v\end{array}\right)$ can be written out in its individual components

$$
\begin{gathered}
\left(\begin{array}{cc}
B & -I \\
I & 0
\end{array}\right)\left(\begin{array}{l}
u \\
v
\end{array}\right)=\lambda\left(\begin{array}{l}
u \\
v
\end{array}\right) \\
B u-v=\lambda u \\
u=\lambda v
\end{gathered}
$$

Subtitling (20) into (19) we find

$$
\left(\lambda B-\lambda^{2}-1\right) v=0 \text { Or } B v=\left(\lambda+\frac{1}{\lambda}\right) v
$$

The latter equation implies that $v$ is the Eigen vector of $B$ with $\lambda+\lambda^{-1}$ the corresponding Eigen values of the tridiagonal matrix $B$

$$
\left(\lambda+\frac{1}{\lambda}\right)=2\left(1-r^{2}+r^{2} \cos \frac{\pi k}{n}\right) \mathrm{k}=1,2 . \mathrm{N}-1
$$

Multiplying both sides of equation (22) by $\lambda$ leads to a quadratic equation for the Eigen values let $a_{k}=1-r^{2}+$ $r^{2} \cos \frac{\pi k}{n}$

$\lambda^{2}-2 a_{k} \lambda+1=0$ Where $1-2 r^{2}<a_{k}<1$ Each pair of solutions to this $n-1$ quadratic equation namely

$\lambda_{k}=a_{k} \pm \sqrt{a_{k}^{2}-1}$ Yields two Eigen values of matrix $\mathrm{C}$

If $a_{k}<-1$ then one of the two eigenvalues will be both $<-1$, which means that the linear iterative system has an exponentially growing mode If $a_{k}<1$, then the Eigen values are complex number of modules 1 , indicated stability of the matrix C [7] that

Therefore in view of $1-2 r^{2}<a_{k}<1$ we should require

$$
r=\frac{c k}{h}<1 \text { Or } k<\frac{h}{c}
$$

This places a restriction on the relative sizes of the time and space steps. We conclude that the numerical scheme is conditionally stable. The stability criterion (23) is known as the Courant condition, The Courant condition requires that the mesh slope, which is defined to be the ratio of the space step size to the time step size, namely $h / k$, must be strictly greater than the characteristic slope $\mathrm{c}$

\subsubsection{Stability of the Explicit Method with Derivative Boundary Condition}

The difference equation that we will use to time stepping the numerical scheme

$$
\begin{gathered}
u_{x}(1, n)=0 \text { Left boundary } j=1, n=1, \ldots, M \\
u_{x,}(N, n)=0 \text { Right boundary } j=N, n=1, \ldots, M \\
u_{j+1, n}=u_{j-1, n} \text { Derivative boundary for } j=0 \\
u_{j-1, n}=u_{j+1, n} \text { Derivative boundary for } j=N \\
u_{j, 1}=f_{j} \text { Row } 1 j=2, \ldots, N-1 n=1
\end{gathered}
$$

$u_{j, 2}=f_{j}+g_{j} k+\frac{r^{2}}{2}\left(f_{j+1}-2 f_{j}+f_{j-1}\right)$ Row $2 j=2, N-1$ $\mathrm{n}=2$

$u_{j, n+1}=\left(2-2 r^{2}\right) u_{j, n}+r^{2}\left(u_{j+1, n}+u_{j-1, n}\right)-u_{j, n-1}$

We can eliminate the fictitious value $u_{j-1, n}$ we see that $u_{j-1, n}=u_{j+1, n}$ from (16), which can be used in (21) to arrive at a modified scheme for the boundary point $u_{0, n+1}$

$$
u_{j, n+1}=\left(2-2 r^{2}\right) u_{j, n}+2 r^{2} u_{j+1, n}-u_{j, n-1} \text { For } j=0
$$

Similarly, if it applied at $x=N$ is discretized by using (31) we have

$u_{j, n+1}=\left(2-2 r^{2}\right) u_{j, n}+2 r^{2} u_{j-1, n}-u_{j, n-1}$ For $j=N$

The modification of the scheme at the boundary is also required for the special formula for the first time step Then we have the explicit scheme with derivative boundary is

$$
\begin{gathered}
u_{j, n+1}=\left(2-2 r^{2}\right) u_{j, n}+2 r^{2} u_{j+1, n}-u_{j, n-1} \text { For } j=0 \\
u_{j, n+1}=\left(2-2 r^{2}\right) u_{j, n}+r^{2}\left(u_{j+1, n}+u_{j-1, n}\right)-u_{j, n-1} j= \\
1, \ldots, N_{x}-1 \\
u_{j, n+1}=\left(2-2 r^{2}\right) u_{j, n}+2 r^{2} u_{j-1, n}-u_{j, n-1} \text { For } j=N x
\end{gathered}
$$

Its matrix form is as follows

$$
\begin{aligned}
& \bar{u}_{n+1}=A \bar{u}_{n}+\vec{d}+b_{i}
\end{aligned}
$$

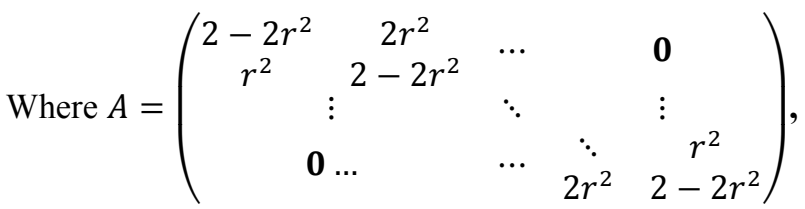




$$
\begin{gathered}
\bar{u}_{n}=\left(\begin{array}{c}
u_{1, n} \\
\vdots \\
u_{j, n}-1
\end{array}\right) \\
\vec{d}=\left(\begin{array}{c}
u_{j, 1} \\
u_{j, 1} \\
\vdots \\
\vdots \\
u_{j, 1}
\end{array}\right)=\left(\begin{array}{c}
f_{j} \\
f_{j}+g_{j} k+\frac{r^{2}}{2}\left(f_{j+1}-2 f_{j}+f_{j-1}\right) \\
\vdots \\
\vdots \\
f_{j}+g_{j} k+\frac{r^{2}}{2}\left(f_{j+1}-2 f_{j}+f_{j-1}\right) \\
\bar{u}_{n+1}=A \bar{u}_{n}+\vec{c} \text { where } \vec{c}=\vec{d}+\vec{b}
\end{array}\right)
\end{gathered}
$$

This is significant result in that it shows that the vector $\vec{c}$ does not affect stability

For stability $p(A) \leq 1$. Consider the matrix $A=$

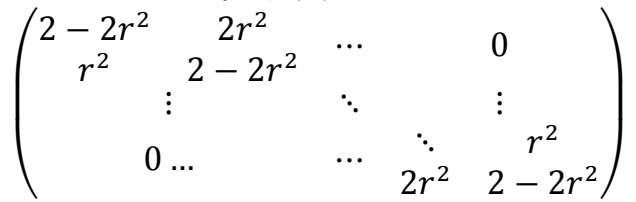

The disks for row 2 to $N-1$ have center $2-2 r^{2}$ and radii $2 r^{2}$ so the eigen value lies in $2-2 r^{2}-2 r^{2} \leq \lambda \leq 2-$ $2 r^{2}+2 r^{2}$

$$
2-4 r^{2} \leq \lambda \leq 2
$$

$2-4 r^{2} \geq-1$ which implies that $r \leq \frac{\sqrt{3}}{2}$

Consider the first and last row with center $2-2 r^{2}$ and radii $2 r^{2}$ The disk is

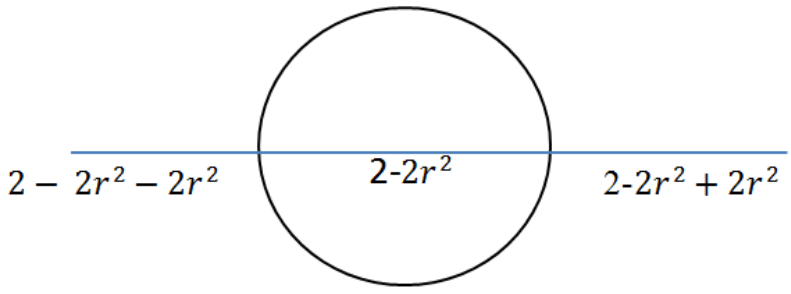

$P(A) \leq 1$ If $2-4 r^{2} \geq-1$ and 2 so the explicit scheme is stable for $r \leq \frac{\sqrt{3}}{4}$

This shows that with derivative boundary condition the explicit scheme stability depends on the values of $r$.

\section{Numerical Experiment}

Numerical examples are presented to verify stability and convergence of the method

Example 1 Use the explicit scheme to solve the one dimensional wave equation

$$
u_{t t}=4 u_{x x} 0 \leq x \leq 1,0 \leq t \leq 1
$$

$u_{x}(0, t)=\cos (2 t), u_{x}(\pi, t)=-\cos (2 t)$ and $u(x, 0)=$ $\sin (x), u_{t}(x, 0)=0$

Example 2. Use the explicit scheme to solve the one dimensional wave equation

$u_{t t}=4 u_{x x} \quad$ For $\quad x \in[0, L] t \in[0, T]$ With boundary condition $u(0, t)=u(L, t)=0 \quad$ Initial distribution is $u(x, 0)=\sin (\pi x)$ and $u_{t}(x, 0)=0$

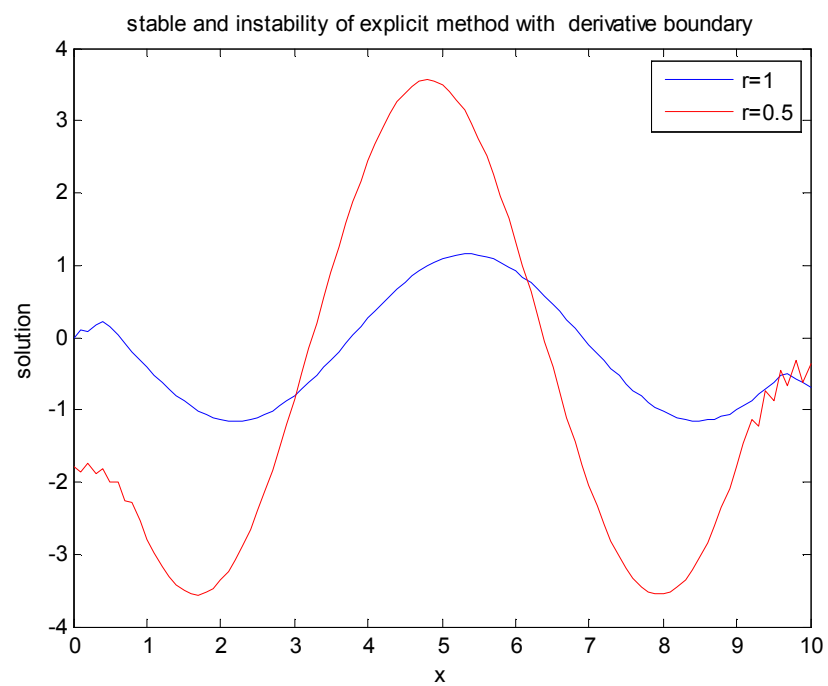

Figure 2. Stability of explicit method for $r=0.5, k=0.05$ and $h=0.1$ at $t_{11}=0.5$ And it's instability for $r=1 k=0.25$ and. $h=0.5$ at $t_{11}=0.25$.

Table 1. Maximum absolute error of example 1 for $r=0.75$ explicit method at different time $t$.

\begin{tabular}{llll}
\hline Time step & $\Delta \boldsymbol{t}$ & $\boldsymbol{t}$ & Explicit Method \\
\hline 2 & 0.0475 & 0.0475 & 0.8254 \\
7 & 0.0475 & 0.2850 & 0.0455 \\
11 & 0.0475 & 0.475 & 5.4487 \\
15 & 0.0475 & 0.665 & 10.2516 \\
19 & 0.0475 & .0 .855 & 18.4482 \\
\hline
\end{tabular}

Table 2. Maximum absolute error of example 1 for $r=1.05$ Explicit method at different time.

\begin{tabular}{llll}
\hline Time step & $\Delta \boldsymbol{t}$ & $\boldsymbol{t}$ & Explicit Method \\
\hline 2 & 0.0525 & 0.0525 & 0.7662 \\
7 & 0.0525 & 0.315 & 2.1107 \\
11 & 0.0525 & 0.525 & 3.7374 \\
15 & 0.0525 & 0.7350 & 6.2255 \\
19 & 0.0525 & .0 .9450 & 32.2921 \\
\hline
\end{tabular}

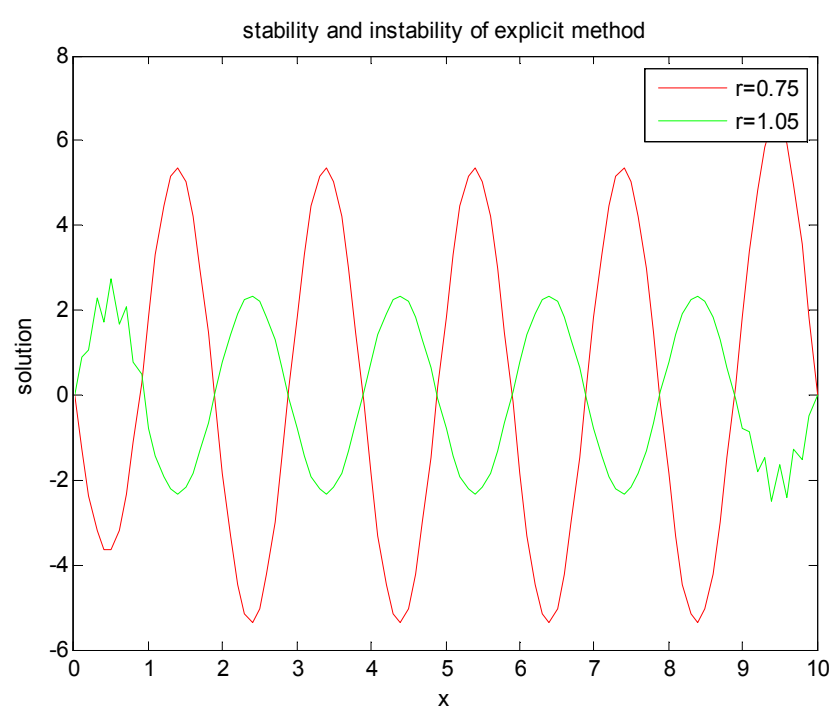

Figure 3. Stability of explicit method for $r=0.75, k=0.0475$ and $h=$ 0.1 at $t_{11}=0.475$ and it's instability for $r=1.05 k=0.0525$ and. $h=0.1$ at 
$t_{11}=0.525$

\section{Discussion and Conclusion}

\subsection{Discussion}

I used one dimensional wave equation by considering the space domain to have a length of $\mathrm{M}$ that is $u_{t t}=c^{2} u_{x x}+$ $f(x, t)$ with drichelet and derivative boundary conditions. The explicit finite differences were considered. To determine its stability by Von Neumann stability condition and Eigen value of tridiagonal matrix obtained from discretized scheme of the equation was used to develop the analysis. To produce values for the problem variable finite difference mesh point were used for the space domain. A mat lab code was written for explicit methods with drichlet and derivative boundary condition

The numerical scheme is said to be stable when an error is introduced at certain stage, then remains bounded as time approaches infinity. It so happen that the error propagates in the same way as the problem variable, so an unstable process can be observed by the solution growing beyond any bounds. With small $r$ the method performed well. The explicit scheme has an advantage that it is easy to set up, and disadvantage that it is unstable for $r$ greater than one with drichilet and unstable for $r \leq \frac{\sqrt{3}}{2}$ with derivative boundary

\subsection{Conclusion}

This study has considered the explicit finite difference schemes for solving one dimensional time dependent wave equation with drichlet and Neumann boundary conditions. The difference schemes are derived. Using Lax Equivalence Theorem convergence of the method was described by testing consistency and stability of the methods. Stability was discussed by using Gerschgorin's Theorem and Von Neumann stability condition. And the stability of the Explict method is shown by the table below

Table 3. Summary of the finding.

\begin{tabular}{lll}
\hline $\boldsymbol{u}_{t t}=\boldsymbol{c}^{2} \boldsymbol{u}_{\boldsymbol{x}}+\boldsymbol{f}(\boldsymbol{x}, \boldsymbol{t})$ & Matrix method and Fourier method & \\
\hline Methods & Drichilet & Derivative \\
\hline Explicit scheme & stable $r \leq 1$ & conditionally stable $r \leq \sqrt{3} / 2$ \\
\hline
\end{tabular}

In the above table the Drichilet boundary condition are $u(0, t)=u(L, t)=0$ and the diivative boundary condition are $u(x, t)=f(x), u_{x}(x, t)=g(x) 1$ is the critical value such that for $r \leq 1$ scheme is stable.

A systematic study was applied to the two test numerical problems and the schemes have been successfully applied. The performance of the schemes for the considered problems was measured by calculating the error.

\section{References}

[1] AUTUMN (2009) the eigen vaue of the tridiagonal matrix.

[2] D. M. Causon; Professor C. G. Mingham (2010). Introductory Finite Differences for PDEs.

[3] Harwinder Kaur (2012). MSc. Thesis on Numerical Solutions of Some Differantial Equations Using B-Spline Collection Method. School of Mathematics and Computer Applications, Thapar University.

[4] J. W. Thomas (1998) numerical partia equation: finite difference methods.

[5] Michael T. Heath (2002). Scientific Computing: An Introductotry Survey Chapter 11- Partial DifferantialEquations.

[6] Michael P. Lamoureux (2006). The mathematics of PDEs and the wave equation.

[7] 2012 Peter J. Olver Vibration and Diffusion in OneDimensional Media.
[8] 2012. Rishu SinglaThesis paper of finite difference equation for parabolic equationHans De and Paul Ullrich (2007-2009). Introductionto Computational PDEs Department of Applied Mathematics University of Waterloo.

[9] Randall J. Leveque (2006). A Finite difference Methods for Differrential Equations. AMath 585, Winter Quarter 2006 University of Washington.

[10] Rishu Singla (2012). Numerical solution of Some Parabolic Partial Differantial Equations Using Finite Difference Methods.

[11] S. Larsson and V. Theme. Partial Differential Equations with Numerical Methods, volume 45 of Texts in Applied Mathematics. Springer, 2003.

[12] Abel Kurura T. and Charles Otieno N. (2018) Stability and Consistency Analysis for Central Difference Scheme for Advection Diffusion Partial Differential Equation Kisii university, Kenya.

[13] Doyo K. and Gofe G. (2016). Convergence Rates of Finite Difference Schemes for the Diffusion Equation with Neumann Boundary Conditions. American Journal of Computational and Applied Mathematics, 6 (2): 92102.

[14] Azad, T. M. A. K., M. Begum and L. S. Andallah. (2015). An explicit finite difference scheme for advection diffusion equation (2015). Jahangirnagar J. Mathematics and Mathematical Sciences 24: 2219-5823.

[15] Chan, T. F. (1984). Stability analysis of finite difference schemes for the advection diffusion equation. SIAM J. Numer. Anal. 21: 272-284. 